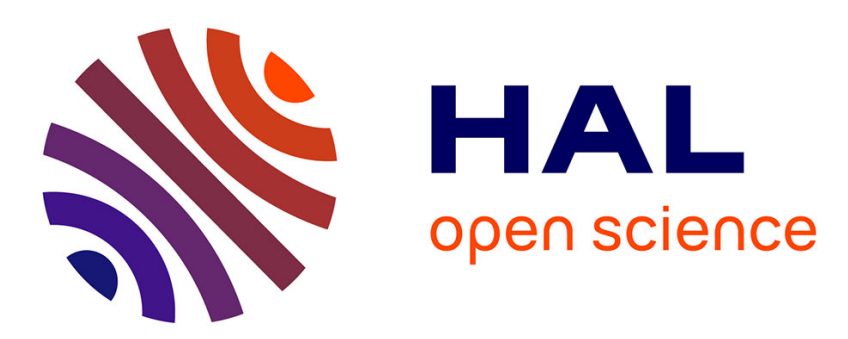

\title{
Le témoin en scène. Sur la temporalité de la figure du témoin dans le tableau
}

Giuseppe Di Liberti

\section{To cite this version:}

Giuseppe Di Liberti. Le témoin en scène. Sur la temporalité de la figure du témoin dans le tableau. Thomas Kisser. Bild und Zeit. Temporalität in Bildender Kunst und Kunsttheorie seit 1800, Fink Verlag, pp.49-63, 2011, 978-3-7705-4806-4. hal-01429334

\section{HAL Id: hal-01429334 https://hal.science/hal-01429334}

Submitted on 5 Jul 2018

HAL is a multi-disciplinary open access archive for the deposit and dissemination of scientific research documents, whether they are published or not. The documents may come from teaching and research institutions in France or abroad, or from public or private research centers.
L'archive ouverte pluridisciplinaire HAL, est destinée au dépôt et à la diffusion de documents scientifiques de niveau recherche, publiés ou non, émanant des établissements d'enseignement et de recherche français ou étrangers, des laboratoires publics ou privés. 
Giuseppe Di Liberti, «Le témoin en scène. Réflexions sur la temporalité du témoignage en peinture », Thomas Kisser. Bild und Zeit. Temporalität in Bildender Kunst und Kunsttheorie um 1800 und seitdem, Fink Verlag, pp.49-63, 2011.

\section{PREPRINT VERSION}

\section{Giuseppe Di Liberti}

Le témoin en scène.

Sur la temporalité de la figure du témoin dans le tableau.

Les trois derniers vers du poème de Celan Aschenglorie disent: "Personne/témoigne pour/le témoin $»^{\mathrm{i}}$. Il y a comme quelque chose de paradoxal derrière ces motsii.

Le paradoxe réside peut-être dans le fait qu'ils se situent exactement sur le seuil entre espace poétique et réalité. Dans la réalité, l'affirmation de Celan est correcte. On ne peut pas avoir des témoins du témoin. Imaginez vous par exemple quelle valeur pourrait avoir dans un tribunal le témoignage de quelqu'un qui déclare avoir vu quelqu'un d'autre voir quelque chose, sans être lui-même témoin de l'événement. En d'autres termes, témoigner est un acte toujours radicalement singulier et fortement localisé. Dans la réalité, le témoignage est toujours une action en deux temps : l'être témoin de quelque chose, donc un acte dépendant d'un autre événement, sans que cette dépendance crée un rapport de cause à effet; le témoigner devant quelqu'un d'avoir été présent à un événement en décrivant l'événement. Dans ce deuxième temps, le témoin demande un acte de foi : parce qu'il est proche de la réalité de l'événement, on lui demande de valider lui-même l'authenticité de ses mots (la célèbre formule de serment avant une déposition dans les procès)iii. Donc il démontre et décrit à la fois l'événement.

Mais qu'est-ce qui arrive quand cet acte a lieu dans un espace poétique qui réclame un lecteur ? Quelque chose arrive. Il arrive que le lecteur devienne témoin d'un témoignage. Ce n'est pas juste un acte de foi : il partage les conventions du témoignage, l'actualise. Il est témoin du témoin et le légitime, ce qu'il ne peut pas faire dans la réalité. Le lecteur doit reconnaître dans le parlant le témoin d'un événement. Car le témoin n'est pas complètement conscient de ce qui se passe, dans une certaine mesure, il est innocent et involontaire : sa dépendance de l'événement est fortuite. Il n'est pas un spectateur, car il n'a pas choisi de regarder ce qu'il a vu, mais surtout parce que ce qu'il a eu devant ses 
yeux n'est pas un spectacle. L'événement n'était pas adressé à lui. Il était tout simplement là, seulement une présence fortuite. Mais nous demandant de lui prêter de la foi, le témoin, sur le seuil entre réel et fictif, nous demande ainsi de reconnaître ce seuil, où la différence entre la vérité de la réalité et la vérité de la fiction est faible, mais où se cache peut-être le sens même du témoignage. Et la question propre à une éthique du témoignage, donc de l'autre comme fondement pour le témoin, devient aussi une question esthétique, car reconnaître ce seuil signifie aussi reconnaître l'acte de construction d'un espace fictif.

Voici alors le paradoxe de ces trois vers de Celan : être à la fois vrais et faux par rapport au lieu d'où le parlant nous parle, avoir une structure qui ressemble quelque part au paradoxe du menteur. Il y a un implicite jeu d'inversion, un peu comme dans le petit recueil d'aphorismes Contre-jouriv.

Mais cette oscillation change ultérieurement quand on passe aux images. Les deux temps du témoignage coïncident. Le témoin en scène à la fois assiste (démontre) et présente (décrit) son témoignage. Il énonce sa vérité - le fait - comme la vérité de l'événement. Et il rend notre présence de spectateurs indispensable. Le témoin devient ici un symptôme de la différence entre arts du temps et arts de l'espace pour venir la réactiver. L'acte du témoigner se condense et provoque une réduction de l'événement à l'instant actualisé. «Ce que je vous montre est vrai, puisque je suis là, et vous le voyez ». La seule fonction est de dire le fait. Et légitimer le fait grâce à et en face de la présence du spectateur. "Je suis là » et ce "là" est à la fois le lieu historique de l'événement et le lieu fictif du tableau [fig. 1 : Gerard David, Nativité, c. 1490, 76,5 x 56 cm, Museum of Fine Arts, Budapest, détail. La figure du témoin est identifiée avec l'autoportrait du peintre]. Et à cette déclaration de présence, le spectateur peut répondre : «J'aurais pu être à sa place $»$.

C'est déjà pour cette simple présence que dans l'image, le témoin peut renvoyer à une dimension figurale, en nous demandant de devenir nous-mêmes témoins de l'acte de construction de l'image qui se répète devant nos yeux, car les faits sont les faits construits par le tableau.

Donc contre-jour nous sommes passés aux images. Il suffirait de feuilleter un manuel quelconque d'histoire de l'art bien illustré, pour tomber sur une myriade d'assistants dont la fonction prééminente est celle de regarder. Nous pourrions même faire une liste de tous 
ceux qui assistent à la scène, en déterminant à tous les coups des fonctions différentes : les anges, les fidèles, les commanditaires d'un martyre ou d'un message, les convives, les artistes qui se mettent en scène pour assister à l'événement qu'ils représenteront après [fig. 2: Albrecht Dürer, Le Martyre de 10000, 1508, 99 x 87 cm, Kunsthistorisches Museum, Wien]. De cette première et brève promenade dans les images, probablement nous pourrions déduire seulement une chose presque banale : il y a des assistants là où il y a un événement - nativité, annonciation... - qui doit montrer sa validité et attend notre validation en tant que spectateurs.

Mais même en considérant la longue liste des assistants possibles, il faut reconnaître qu'il n'est pas évident d'isoler une Figure qui remplisse exclusivement la fonction de témoin. Nous pouvons parler de toutes ces figures comme figures d'assistants, plus ou moins en choeur, qui prennent part à la narration et qui ont presque toujours une fonction iconographique précise, qui sont en scène et participent à la narration. En revanche, le témoin, si toutefois il peut - à certaines conditions - renforcer le dispositif narratif, est à la fois en scène et hors scène, au seuil de la scène [fig. 3 : Maître de Moulins, Nativité, c. 1480, 55x71 cm, Musée Rolin, Autun]. Pour cette raison, il y a souvent des dispositifs qui accompagnant le témoin pour l'isoler : des fenêtres, des niches, des ruines qui mettent le témoin en marge. Dans un certain sens, le témoin arrête le temps de la narration et dévoile que quelque chose arrive brutalement dans - et parfois contre - la représentation. Effet de surprise. Comme au théâtre les machinistes habillés de noir lors d'un changement de décor précipité, le témoin contrevient à la mise en scène, enlève de scène et dévoile le fait en tant que tel.

Pour reprendre une expression de Blumenberg, le témoin est dans une position de «Quasi-Außerweltlichkeit »v, alors que, si le témoin reste même un spectateur, il sera proche de la métaphore lucrétienne du spectateur d'un naufrage. Mais par rapport au spectateur de Lucrèce, le témoin représenté dans le tableau est neutre face aux événements tant sur le plan existentiel que sur le plan théorétique, et il nous renvoie toute réflexion et prise de conscience des événements. D'une certaine manière, les deux temps du témoignage condensés par le témoin représenté reviennent dans notre expérience de spectateurs : à travers la médiation du témoin et devant le tableau, nous devenons des témoins et nous prenons en charge la valeur et la validité du témoignage. Mais qu'est-ce que représente enfin le témoin dans le tableau ? Il reproduit en scène une distance, encore 
une fois, paradoxale. La distance et la proximité qu'il représente par rapport à l'événement est la même de notre distance et de notre proximité au tableau en tant que représentation de l'événement, en tant que présentation et construction des faits. Il montre le fait que l'événement a eu lieu et il nous pousse à reconnaître la légitimité de ce fait. Dans ce sens, le témoin est une figure théorique: il représente surtout une forme de l'expérience esthétique comme forme d'expérience de connaissance.

Il faudrait peut-être éclaircir, même brièvement, le rapport entre fait et événement, en reprenant, très partiellement, une distinction qui, à partir de Wittgenstein et de Russell, en passant par Austin et Strawson pour arriver à Davidson, a été l'objet d'un débat, parfois controversé, dans la logique, dans la métaphysique et surtout dans les théories de la vérité contemporaines $^{\mathrm{vi}}$. Justement un célèbre symposium entre Austin et Strawson permet de

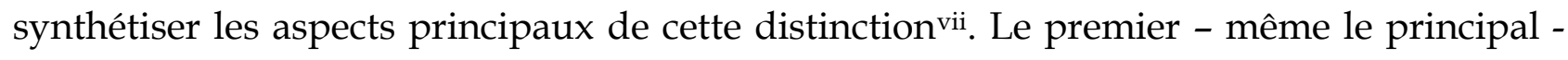
est si nous pouvons considérer les faits comme choses du monde. Austin pense que oui, en employant le mot "fait" pour parler des situations, des événements historiques, des choses, car pour Austin si une affirmation (statement) est vraie, elle est vraie parce qu'elle correspond aux faits et donc, pour lui, les faits font partie de notre monde avec les objets et les événements. C'est justement sur ce point que Strawson avance sa critiqueviii. Pour lui, Austin commet l'erreur de mélanger faits et événements qu'il faut, au contraire, garder distincts car «Events can be dated and things can be located. But the facts which statements (when true) state can be neither dated or located. (Nor can the statements, though the making of them can be.) Are they included in the world? »ix. Donc pour Strawson les faits ne sont pas des choses auxquelles les affirmations se réfèrent, mais plutôt ce que les affirmations vraies affirment («Facts are what statements (when true) state ; they are not what statements are about $\left.»^{x}\right)$. En synthétisant la position de Strawson aussi dans ses essais suivants, on peut dire que les faits font partie de nos outils pour expliquer et mettre en relation nos affirmations sur le monde. D'ailleurs, la nécessité de distinguer faits et événements présentée par Strawson avait été déjà avancée par Ramsey en 1927, toujours dans un symposium de l'Aristotelian Society tenu avec Moorexi, pour être ensuite gardée - ainsi comme l'inévitable critique à Austin - par plusieurs philosophes analytiques, parmi lesquels Chisholm ${ }^{x i i}$ ou Vendlerxii. Et nous arrivons jusqu'à Davidson, pour lequel cette distinction est même une condition nécessaire pour penser une ontologie des événements ${ }^{\mathrm{xiv}}$ comme entités particulières, c'est-à-dire entités uniques localisées dans 
l'espace et dans le temps.

En guise de synthèse, nous pouvons donc dire que plusieurs faits différents peuvent correspondre à un événement historique particulier, par exemple la mort de César. Avant tout, le fait que tel événement ait eu lieu. Ensuite, le fait que César ait été poignardé à mort, le fait que Brutus ait poignardé César, le fait que Brutus ait poignardé César violemment, le fait que Brutus ait poignardé César en le tuant, etc. Un événement, stable dans l'espace et le temps - la mort de César au 44 av. J.-C. à Rome -, peut être décrit et redécrit par beaucoup d'affirmations qui établissent des faits, qui ont un caractère abstrait et atemporel (le fait que Brutus ait poignardé César est un fait hier comme aujourd'hui, à Rome comme à Paris) et souvent partiel (dans le sens qu'ils peuvent présenter des termes singuliers ou des propriétés différents pour faire référence au même événement ou au même objet). Les faits donc nous permettent d'expliquer les relations entre nos assertions. "Voyons les faits », comme peut le dire le détective d'un polar, signifie en général faire une liste des affirmations - des faits - dans le but d'établir des relations ${ }^{\mathrm{xv}}$. Les faits sont alors plus dans notre discours sur le monde que dans le monde même et à travers les faits nous expliquons notre version du monde. Pour cette raison, le témoin nous donne des faits et non des événements, sa version dont nous devons constater la cohérence et la correspondance.

Pour passer maintenant aux images et au rôle du témoin, nous pouvons reformuler la question dans les termes qui suivent. Dans un monde peuplé par des objets et des événements, il n'y a pas de place pour les faits. Les faits ne sont pas dans le monde, mais dans nos constructions sur le monde. Ils ne sont pas non plus des parties des événements mais plutôt des versions - souvent partielles - pour expliquer nos affirmations sur les événements. Reste le problème de comprendre la relation de correspondance entre les faits et les événements. L'hypothèse que je voudrais ici poursuivre - et qui est en bonne partie influencée par la réflexion de Nelson Goodman - est de mettre entre parenthèse les problèmes de vérité en termes de correspondance au monde, en disant que la relation entre faits et événements n'est pas invariable, mais qu'elle est conditionnée par le système symbolique dans lequel le fait est construit. L'adoption de cette hypothèse ne signifie pas en revanche opter tout court pour une forme de relativisme radical, car on ne nie pas l'existence d'un monde d'objets et d'événements : on affirme tout simplement que le rapport entre l'événement et le fait établi par une affirmation vraie est différent du rapport 
entre l'événement et le fait présenté par une image picturalexvi.

Dans cette perspective, le fait construit et présenté dans le tableau se donne comme relativement indépendant de l'événement et comme tel il demande d'être considéré. Dans l'image, l'événement sera alors une certaine donnée historique - d'une histoire réelle ou imaginaire -, qui a lieu dans l'histoire (ou dans une histoire), un fragment d'espace-temps, une action placée, une transition d'un état de choses à un autre. Le fait sera ce qui se produit ainsi et ainsi, en constituant le matériel spécifique d'une image particulière et construit par l'image une version du monde. Mais le fait n'est pas déjà l'image, mais plutôt le matériel pour l'image, le point de vue que l'image met en jeu.

Pour observer certains aspects de cette mise en jeu opérée à travers la figure du témoin, je voudrais maintenant considérer quelques tableaux d'annonciation, que je prends ici comme cas exemplaire des questions qui existent entre fait, image et témoin. J'ai cherché à mettre à l'épreuve ce thème en suivant l'itinéraire tracé par Daniel Arasse dans son travail consacré aux annonciations italiennes. L'annonciation en tant qu'événement est un événement invisible, presque sans témoins, secret. Dans l'introduction à son livre consacré aux annonciations, Arasse présente deux textes, que nous pourrions voir comme les deux références constantes pour les représentations figuratives de l'Annonciation. Le premier est un passage de l'Évangile de Luc qui raconte l'épisode et qu'Arasse commente ainsi: «Pur dialogue, récit d'un échange verbal, ce texte se présente comme une épure narrative, dépourvue de toute précision sur les circonstances de l'événement. Ce laconisme descriptif, appuyé par les enrichissements narratifs dont cette version originelle fera l'objet, laissera d'ailleurs aux peintres une grande liberté dans l'invention de ces circonstances, qu'il s'agisse du cadre dans lequel a lieu l'Annonciation, la maison de Marie, de l'activité de cette dernière au moment où Gabriel entre (par où?) chez elle, de l'apparence des personnages, etc.»xvii. Le deuxième texte est le sermon De Triplici Cristi Nativitate de Bernardin de Sienne, riche en oxymores exprimant le mystère et de l'irreprésentabilité de l'Incarnation. J'en cite juste quelques phrases: l'Incarnation est le moment où «l'éternité vient dans le temps, l'immensité dans la mesure [...], l'infigurable dans la figure, l'inénarrable dans le discours, l'inexplicable dans la parole, l'incirconscriptible dans le lieu, l'invisible dans la vision...»xviii. En un certain sens, dans ces deux passages, nous avons respectivement l'événement et les faits (ou au moins des faits). À l'événement “Annonciation" auquel le récit de Luc se réfère, correspondent des 
faits de l'expérience ou mieux, dans le cas spécifique, des limites sensibles et intelligibles de l'expérience même. Distinguer fait et événement ne signifie pas, dans l'image comme dans une nominalisation, description ou représentation, choisir entre fait et événement, qui peuvent avoir, d'autre part, un certain rapport de dépendance. Distinguer fait et événement signifie scander ce processus cognitif qui va de l'événement à l'expérience et de l'expérience à l'image ou à la description verbale. Nous pourrions dire que chaque image singulière, ainsi comme chaque proposition singulière, se forme sur une construction des faits et sont les faits qui constituent le matériel de l'image et encore les faits qui nous permettent une analyse de l'image. Revenons en donc au témoin.

Quel rapport a le témoin avec le fait? Et quelle fonction a-t-il dans la présentation du fait? Nous pourrions tenter une réponse en disant que la figure du témoin est un des dispositifs possibles qui fonctionnent à l'intérieur de l'image, grosso modo, comme la locution "le fait que..." à l'intérieur d'une phrase. La présence du témoin accentue la dimension du fait, médiatise - excusez la répétition - le fait que les choses se sont passées ainsi et ainsi.

Maintenant je passe le mot aux images. La première est une Annonciation du 1335-50 attribuée à Barna de Sienne (ou à Lippo Memmi), fresque qui se trouve à la Collegiata de San Gimignano [fig. 4 : Barna de Sienne, Annonciation, 1335-50 c., Collegiata de San Gimignano, dét.]. D'après mes connaissances, elle fait partie des premières Annonciations dans la culture figurative italienne dans lesquelles il y a un témoin, la jeune femme amie de Marie qui derrière le mur tend l'oreille pour saisir la conversation. Arasse poursuit: «Tel que l'a conçu "Barna", le lieu de la rencontre est artificiel, affirmé et même exhibé comme tel : avec sa moulure, le socle de la maison de Marie constitue un véritable lieu scénique, la "mansion" de la Vierge telle qu'elle aurait pu se présenter dans une représentation théâtrale contemporaine [...]. En approchant théâtralement son oreille de la paroi pour tenter d'entendre le dialogue évangélique, celle-ci montre au spectateur que "quelque chose" a commencé de se dire et elle est aussi un relais spirituel qui invite le fidèle à prononcer, intérieurement ou à haute voix, l'Ave Maria»xix. Cet aspect de médiation est à mettre en relation avec un autre élément de modernité de la fresque que Arasse ne manque pas de remarquer: le fait que Marie se tourne plus vers le spectateur que vers Gabriele. Autre aspect souligné par Arasse ${ }^{x x}$ est la reprise de la part de Barna du schéma - même si renversé - de l'Annonciation à Anne de Giotto à la Chapelle des 
Scrovegni. Je voudrais seulement ajouter que cette scène est la troisième du cycle entier de la Chapelle, précédée seulement par l'expulsion de Joachim du Temple et de Joachim avec les bergers, et que cette scène constitue en effet le début de la narration évangélique (même s'il s'agit des évangiles apocryphes), ce qui pourrait constituer une raison pour poser un témoin en scène, en tant qu'élément de validité du récit. Tandis que la plupart des Annonciations précédant ou suivant immédiatement celle de Barna semblent montrer surtout une attention pour le dialogue en cours, autrement dit, pour l'événement dans sa durée. L'Annonciation de Barna semble vouloir saisir un instant précis de ce dialogue, un instant d'interruption où Marie regarde ailleurs, vers le spectateur.

Après celle de Barna, les Annonciations avec témoin seront de plus en plus fréquentes. Luc même sera représenté comme témoin (voir par exemple l'Annonciation de Benedetto Bonfigli, 1450-1453, Pérouse, Galerie Nationale de 1'Ombrie) qui pourrait être prise comme exemple d'une relation entre événement et fait. Mais peut-être il nous montre aussi que dans l'espace de l'image il y a seulement des faits dans leur autonomie de construction. Aussi dans ce cas, le témoin est porteur d'une version et d'un temps (le temps du récit qu'il écrit, l'instant où il regarde la rencontre entre Maria et Gabriele). Encore deux exemples: 1'Annonciation de Spello de Pinturicchio [fig. 5 : Pinturicchio, Annonciation, fresque, c. 1500-1501, Santa Maria Maggiore, Chapelle Baglioni, Spello, dét.] dans laquelle Pinturicchio met son portrait dans un cadre accroché dans la domuncula de Marie et à propos duquel Arasse écrit, en citant Louis Marin: «Ce retrait permet de "dresser la scène" de l'Annonciation et suggère que ce qui est représenté là, plus qu'un simple "récit iconique de l'historia" constitue sa "mise en représentation"»xxi. Et encore une autre Annonciation célèbre, plus tardive, celle de Lorenzo Lotto où le témoin est, comme tout le monde le sait, un chat qui bondit à travers la chambre [fig. 6: Lorenzo Lotto, Annonciation, c. 1527, 166x114 cm, Pinacoteca Comunale, Recanati]. Ces deux exemples nous montrent clairement les effets temporels que les figures de témoin exercent sur le tableau. L'autoportrait de Pinturicchio - avec le paysage très humain sur le fond et le rapport d'équilibre qu'il a avec la figure de Dieu le Père -, est une vraie machine de temps : le peintre, en vertu de son art, est présent avant, pendant et après l'événement et il arrive jusqu'à nous pour en témoigner. Si Dieu a créé l'événement, c'est bien au peintre que revient la construction du fait, qui est donc humain, abstrait et intemporel. Et l'effet de cette "machine de temps" est de rendre toujours actuelle l'annonciation, de nous rendre 
possible une légitimation du fait au-delà des limites temporelles de l'événement. Sur l'Annonciation de Lotto, Umberto Eco a écrit: « Combien dure la vision que Marie a de l'ange? Un instant, un clin d'oeil, le temps que le chat emploie à traverser avec un bond la chambre. Vous remarquerez que même ici, pour saisir presque l' “intemporalité" de l'événement, vous devez avoir connu l'expérience du bond d'un chat. [...] Il est certain que s'il y avait une tortue dans le tableau au lieu d'un chat, malgré Zenon, nous sentirions cet instant extatique de Marie comme très long. Le temps du Lotto est un temps signifié. Sur le plan du signifiant nous percevrions toujours dans un seul coup d'oeil tant le chat que la tortue »xxii. Le temps du témoin est toujours un temps signifié, car il introduit dans le tableau un temps vécu singulier qui renvoie à un temps vécu par le spectateur : il nous oblige à reconnaître que le temps du tableau n'est pas le temps de l'événement, mais le temps de la construction d'une version qui pourra être saisie à travers notre expérience individuelle.

Encore un dernier exemple, peut-être le plus extrême, de mise en jeu du témoin : la dernière des Annonciations de Antonello [fig. 7: Antonello da Messina, Vierge de l'Annonciation, Huile sur bois, c. 1475, 45x34 cm, Galleria Regionale della Sicilia Palazzo Abatellis, Palerme] où la figure d'un témoin manque ainsi comme l'ange Gabriel. En revanche, le tableau ne représente pas juste la Vierge, mais l'entier récit de l'annonciation y est condensé, condensation qu'on peut remarquer aussi à travers une comparaison avec les deux autres Annonciations de Antonello, le diptyque conservé à Syracuse et celle de l'Alte Pinakothek de Munich. Marie regarde l'ange Gabriel. Ses mains, son regard, son sourire résument tout le dialogue : la surprise, l'interrogation, la peur, l'acceptation, la joie. Le tableau est un petit format, à l'échelle 1. Il nous oblige à nous rapprocher. Le coin du pupitre touche le cadre, en laissant ouvert l'espace du tableau. La place du témoin est à nous, hors-champ.

Les conclusions que je me permets de proposer sont seulement des points de départ, peut-être pour une recherche plus étendue sur le témoin en peinturexxiii ainsi comme en photographiexxiv et sur le sens du témoin dans les théories de la connaissance, en particulier dans son rapport avec le sens commun ${ }^{\mathrm{xxv}}$.

(1) Du côté $d u$ tableau. La figure du témoin peut être analysée comme une exemplification - parmi d'autres, évidemment - du rapport entre la construction du fait et sa mise en représentation. On pourrait appeler le résultat de ce rapport un "fait pictural", 
c'est-à-dire la version singulière et particulière d'une chose ou d'un événement, version qui constitue le matériel de l'image peinte.

(2) Du côté du spectateur. Dans le tableau, le témoin est une figure de médiation, et pas ou pas seulement - parce qu'il est un spectateur en scène. Nous ne pouvons pas voir ce qu'il voit. Mais sa fonction est de voir, comme la nôtre devant le tableau. Il présente une forme d'expérience esthétique, en nous invitant à faire la même chose. Son temps prend sens à travers notre vécu. Il témoigne, il nous demande donc de valider son témoignage.

(3) Du côté de la théorie. La présence du témoin identifie un regard singulier et surtout une manière de regarder (ou de pas regarder) et une manière de construire l'image, qui n'est rien d'autre, peut-être, que la théorie de l'art que le tableau nous présente. La figure du témoin incarne une théorie précise de la peinture et de la représentation. Le témoin explicite la nécessité d'un échange entre le regard de l'artiste - sa théorie, son contexte, sa version - , l'objet pictural et le regard du spectateur - sa théorie, son contexte, sa version.

\footnotetext{
i «Niemand/ zeugt für den/ Zeugen». Paul Celan, Aschenglorie, in Atemwende, Frankfurt a. M., Suhrkamp, 1967.

ii Je n'entrerai pas dans la question du sens du témoignage dans l'expérience humaine et la poétique de Paul Celan, et j'emploierai ces vers seulement comme point de départ préliminaire pour quelques réflexions sur la temporalité spécifique que la figure du témoin instaure dans certains tableaux. La mise entre parenthèse des aspects éthiques et historiographiques que l'idée du témoin a assumé dans la réflexion du siècle passé, ne signifie pas oublier sa valeur : il signifie plutôt chercher une compréhension plus profonde du concept, en analysant un fragment de la constellation dont il est porteur.

iii Cette division en deux temps et le problème de l'acte de foi peut être bien exemplifié par la réflexion de Droysen sur la nécessité pour l'histoire de séparer le témoin du témoignage en tant que document (voir J. G. Droysen, Historik, Band 1 : Rekonstruktion der ersten vollständigen Fassung der Vorlesungen (1857), Grundriß der Historik in der ersten handschriftlichen (1857/1858) und in der letzen gedruckten Fassunf (1882), Stuttgart-Bad Cannstatt, Frommann-Holzboog, 1977).

iv Cfr. par exemple Paul Celan, Le Méridien \& autres proses, traduit de l'allemand et annoté par Jean Launay, Paris, Seuil, 2002, p. 26: «Enterre la fleur et dépose l'homme sur la tombe» («Vergrabe die Blume und lege den Menschen auf dieses Grab») ; Ibid. p. 28 «Ne te trompe pas: ce n'est pas cette dernière lampe qui donne plus de lumière - c'est l'obscurité qui s'est approfondie» («Täusche dich nicht: nicht diese letze Lampe sendet mehr Licht - das Dunkel rings hat sich in sich selber vertieft») ; Ibid. p. 29 : «Elle tournait le dos à son miroir, car elle détestait la vanité du miroir» («Sie kehrte dem Spiegel den Rüncken, denn sie haßte die Eitelkeit des Spiegels»).

${ }^{v}$ H. Blumenberg, Schiffbruch mit Zuschauer. Paradigma einer Daseinsmetapher, Frankfurt a. M., Suhrkamp, 1979, p.29.

vi Une riche bibliographie à ce sujet est celle de Roberto Casati et Achille Varzi, 50 years of Events. An Annotated Bibliography 1947 to 1997, Bowling Green, Ohio, Philosophy Documentation Center, Bowling Green State University, 1997; on line in Stantford Encyclopedia of Philosophy http://plato.stanford.edu/entries/events/

vii J. L. Austin, P. F. Strawson, D. R. Cousin, "Symposium: Truth", in Proceedings of the Aristotelian Society, Supplementary Volumes, Vol. 24, Physical Research, Ethics and Logic, 1950, pp. 111-172.

viii P. F. Strawson, ibid., p. 135 : « The only plausible candidate for the position of what (in the world) makes the statement true is the fact it states ; but the fact it states is not something in the world. It is not an object ; not even (as some have supposed) a complex object consisting of one or more particular elements
} 
(constituents, parts) and a universal element (constituent, part). I can (perhaps) hand you, or draw a circle round, or time with a stop-watch the things or incidents that are referred to when a statement is made. Statements are about such objects ; but they state facts. Mr. Austin seems to ignore the complete difference of type between, e.g., "fact" " and "thing" ; to talk as if "fact" were just a very general word (with, unfortunately, some misleading features) for "event", "thing", etc., instead of being (as it is) both wholly different from these, and yet the only possible candidate for the desired non-linguistic correlate of "statement" ".

ix Ibid., p. 140.

x Ibid., p. 136.

xi F. P. Ramsey, G. E. Moore, "Symposium: Facts and Propositions", in Proceedings of the Aristotelian Society, Supplementary Volumes, Vol. 7, Mind, Objectivity and Fact, 1927, pp. 153-206. F. P. Ramsey, ibid., p. 156 : «a phrase like "the death of Cesar" can be used in two different ways ; ordinarily, we use it as the description of an event, and we could say that "the death of Caesar" and "the murder of Caesar" were two different descriptions of the same event. But we can also use "the death of Caesar" in a context like "he was aware of the death of Caesar" meaning "he was aware that Caesar had died" ; here (and this is the sort of case which occurs in the discussion of cognition) we cannot regard "the death of Caesar" as the description of an event ; if it were, the whole proposition would be, "There is an event $\mathrm{E}$ of a certain sort, such that he is aware of E", and would be still true if we substituted another description of the same event, e.g., "the murder of Caesar". That is, if his awareness has for its object an event described by "the death of Caesar", then, if he is aware of the death of Caesar, he must also be aware of the murder of Cesar, for they are identical. But, in fact, he could quite well be aware that Caesar had died, without knowing that he had been murdered, so that his awareness must have for its object not merely an event but an event and a character also. The connection between the event which was the death of Caesar and the fact that Caesar died is, in my opinion, this : "That Cesar died" is really an existential proposition, asserting the existence of an event of a certain sort, thus resembling "Italy has a King", which asserts the existence of a man of a certain sort. The event which is of that sort is called the death of Cesar and must no more be confused with the fact that Caesar died, than the King of Italy should be confused with the fact that Italy has a King ».

xii R. M. Chisholm, “Austin's Philosophical Papers", in K. T. Fann (ed.), A Symposium on J. L. Austin, London, Routledge and Kegan Paul, 1979, pp. 101-26.

xiii Z. Vendler, "Facts and Events", in Linguistics in Philosophy, Ithaca, NY, Cornell University Press, 1967, pp. 122-146.

xiv D. Davidson, "Causal Relations", in The Journal of Philosophy, 64, 1967, pp. 691-703 (réédité in Id., Essays on Actions and Events, Oxford, Clarendon Press 1980); Id., "The Individuation of Events", in N. Rescher, ed., Essays in Honor of Carl G. Hempel, Dordrecht, Reidel, 1969, pp. 216-34 (réédité in Id., Essays on Actions and Events, op. cit.); Id., "True to the Facts", in The Journal of Philosophy, Vol. 66, No. 21, Sixty-Sixth Annual Meeting of the American Philosophical Association Eastern Division, Nov. 6, 1969, pp. 748-764.

xv Malgré son emploi des faits non distingués des événements, j'utilise ici un exemple qu'Austin formula toujours dans le symposium sur la vérité. Austin, "Symposium: Truth", in op. cit., p. 117 : " "fact" is only an alternative expression for "true statement". We note that when a detective says "Let's look at the facts" he doesn't crawl round the carpet, but proceeds to utter a string of statements : we even talk of "stating the facts" ».

xvi Dans cette hypothèse, je me permets donc d'accepter les propos de Goodman sur la fabrication des mondes relativement aux œuvres d'art, car la fabrication des faits comme version du monde me semble être une clé de compréhension de l'œuvre en soi. En revanche, je souhaiterais retravailler cette hypothèse à la lumière des réflexions de Donald Davidson, travail qui pose un certain nombre de problèmes dont je ne peux pas discuter ici et qui feront objet d'une recherche ultérieure.

xvii D. Arasse, L'Annonciation italienne. Une histoire de perspective, Paris, Hazan, 1999, p. 10.

xviii Ibid., pp. 11-12.

xix Ibid., p. 95.

xx Ivi.

xxi Ibid., p. 242. Arasse se réfère au texte de Marin Opacité de la peinture. Essais sur la représentation au Quattrocento, Paris, Usher, 1989 (réédition Paris, Éditions de l'École des études en sciences sociales, 2006).

xxii Umberto Eco, "Les sémaphores sous la pluie", in Golem, Dossier Spazialità e testo letterario, avril 2003, on line http:// www.golemindispensabile.ilsole24ore.com/index.php?_idnodo=8702 [traduit de l'italien par G. Di Liberti]. 
xxiii Voir la réflexion de Deleuze sur la fonction-témoin dans la peinture de Bacon (G. Deleuze, Francis Bacon. Logique de la sensation, Paris, La Différence, 1981).

xxiv Voir J.-M. Schaeffer, L'image précaire, Paris, Seuil, 1987, en particulier le $\S 6$ du chapitre 3.

xxv Voir par exemple, le numéro 88 de Philosophie consacré justement au témoignage et en particulier la première partie "Perspectives analytiques: l'épistémologie du témoignage" coordonnée par P. Engel (P. Engel et E. Housset, sous la direction de, "Le témoignage", Philosophie, n 88, Paris, Les Éditions de Minuit, hiver 2005). 